\title{
Swing-Free Transporting of Two-Dimensional Overhead Crane Using Sliding Mode Fuzzy Control
}

\author{
Diantong Liu, Jianqiang Yi, Dongbin Zhao, and Wei Wang
}

\begin{abstract}
An adaptive sliding mode fuzzy control approach is proposed for a two-dimensional overhead crane. System linearization transforms the two-dimensional system to two independent systems: $X$-direction transport system and Y-direction transport system. Both the two systems are with the same dynamic model and both include two subsystems: positioning subsystem and anti-swing subsystem. A sliding mode fuzzy control approach is proposed for both $\mathrm{X}$-direction transport and Y-direction transport, and it combines SMC's robustness and FLC's independence of system model. According to the influences on system dynamic performance, both of the slope of sliding mode surface and the relationship between subsystems are automatically tuned by real time fuzzy inference respectively. The effectiveness of the proposed control is demonstrated by experiments with a two-dimensional prototype overhead crane.
\end{abstract}

\section{INTRODUCTION}

$\mathrm{O}$ verhead crane works as a robot in many places such as workshops and harbors to transport all kinds of massive goods. It is desired for the overhead crane to transport its payloads to the required position as fast and as accurately as possible without collision with other equipments. Moreover, the payload swing angle should be kept as small as possible.

Many works have been done in controlling the overhead crane. Park [1] and Singhose [2] adopted input shaping control method. But the input shaping must be pre-calculated accurately according to the system model. These approaches lacked robustness to external disturbances and couldn't damp residual swing well. Moreover, zero initial condition must be satisfied. Lee [3] and Giua [4] proposed feedback control methods. Besides needing accurate system model and onerous matrix computation, the above methods were greatly affected by system linearization

Manuscript received September 6, 2003.

Diantong Liu is with College of Computer Science, Yantai University, Yantai 264005, China. Phone: 86535-6904991, email: diaton@sina.com, diantong.liu@mail.ia.ac.cn.

Diantong Liu, Jianqiang Yi, Dongbin Zhao, and Wei Wang are with the Key Laboratory of Complex Systems and Intelligence Science, Institute of Automation, Chinese Academy of Sciences, P. O. Box 2728, Beijing, 100080, China. Phone: 8610-82615422; fax: 8610-62658815; e-mail: \{jianqiang.yi, dongbin.zhao,w.wang\}@mail.ia.ac.cn. and system parameters uncertainty.

Fuzzy logic control (FLC) is independent of system model and has some robustness. Lee [5] used fuzzy logic only in anti-swing control and applied position servo control for positioning and swing damping. Hua [6] only studied anti-swing control with fuzzy logic and didn't take positioning control into consideration. Nalley [7] adopted fuzzy logic to both positioning control and swing damping. However, because of the large number of fuzzy rules, it was difficult to set both rules and parameters of the controller only according to experiences.

Sliding mode control (SMC) is a robust design methodology using a systematic scheme based on a sliding mode surface and Lyapunov stability theorem. The main advantage of SMC is that the system uncertainties and external disturbances can be handled under the invariance characteristics of system's sliding mode state with guaranteed system stability. Er [8], Kakoub [9] and Hasanul [10] used the variable structure control (VSC) with sliding modes to control the overhead crane. In [8] and [9], the VSC was used to the positioning control and hoisting control, but another state feedback control scheme must be added for payload swing damping control. In [10], a reference model was defined to track, and the system model must be linearized. All the above VSC methods have difficulties in automatically tuning the relationship between positioning control and anti-swing control.

This paper presents a practical solution to analyze and control the overhead crane. The payload swing and crane motion of two transport directions are considered. Now that SMC is capable of tackling non-linear system with parameter uncertainties and external disturbances, and fuzzy logic control is independent of system model, crane system model is built to analyze system control characteristics without taking external disturbances (such as winds) and system parameters varying (such as different goods) into consideration. A sliding mode fuzzy control algorithm is designed for both X-direction and Y-direction transports of the overhead crane. Combining SMC's robustness and FLC's independence of system model, the proposed control law can guarantee a swing-free transportation.

The remainder of this paper is organized as follows. In 
section 2, the dynamic model of 2-dimensional overhead crane is built, the linearized model is derived and a conclusion is obtained that two-dimensional overhead crane can be divided into two independent transport systems. In section 3, an adaptive fuzzy sliding mode control algorithm is proposed for both $\mathrm{X}$-direction and $\mathrm{Y}$-direction transportations. In section 4 , the proposed algorithm is validated through experiments. Finally, in section 5, conclusions are drawn.

\section{DyNAMic Model OF OVERHEAD CRANE}

In this section, the system description of two-dimensional overhead crane will be given and its dynamic model will be built. Then the model will be transformed by linearization and state feedback to a system that is composed of two transport systems with the same structure. In this way, the system control and its implementation are simplified.

\section{A. System Description}

Figure 1 shows the coordinate system [11] of a two-dimensional overhead crane and its payload. $\mathrm{XYZ}$ is the inertial coordinate system, $M_{X}$ and $M_{Y}$ respectively are the $\mathrm{X}$-direction trolley mass and Y-direction trolley mass including the moment-of-inertia of the gear train and motors. $\theta$ is the swing angle of the payload in XYZ space and it has two components $\theta_{X}$ and $\theta_{Y} \cdot \theta_{X}$ and $\theta_{Y}$ are the swing angle projected on $\mathrm{XZ}$ plane and $\mathrm{YZ}$ plane respectively. Assume the dynamic model has the characteristic that the payload and the trolley are connected by a massless, rigid link.

\section{B. System Dynamics}

According to Lagrangian equation:

$$
\frac{d}{d t}\left(\frac{\partial L}{\partial \dot{q}_{i}}\right)-\frac{\partial L}{\partial q_{i}}=T_{i} \quad(i=1,2,3,4)
$$

where, $L=K-U, K$ is system kinetic energy, $U$ is system potential energy, $q_{\mathrm{i}}$ is generalized coordinate (here is $x, y$, $\theta_{X}$ or $\left.\theta_{Y}\right)$, and $T_{i}$ is external force (here is $f_{X}$ or $\left.f_{Y}\right)\left(T_{3}=T_{4}=0\right)$. The motion equations of the overhead crane system can be obtained with respect to the generalized coordinates $x, y, \theta_{X}$ and $\theta_{Y}$.

$$
\begin{aligned}
& \left(M_{X}+m\right) \ddot{x}+m l \ddot{\theta}_{X} \cos \theta_{X} \cos \theta_{Y}-m l \ddot{\theta}_{Y} \sin \theta_{X} \sin \theta_{Y} \\
& -m l \dot{\theta}_{X}^{2} \sin \theta_{X} \cos \theta_{Y}-m l \dot{\theta}_{Y}^{2} \sin \theta_{X} \cos \theta_{Y} \\
& -2 m l \dot{\theta}_{X} \dot{\theta}_{Y} \cos \theta_{X} \sin \theta_{Y}=f_{X}-D_{X} \dot{x} \\
& \left(M_{Y}+m\right) \ddot{y}+m l \ddot{\theta}_{Y} \cos \theta_{Y}-m l \dot{\theta}_{Y}^{2} \sin \theta_{Y}=f_{Y}-D_{Y} \dot{y} \\
& m l^{2} \ddot{\theta}_{X} \cos ^{2} \theta_{Y}+m l \ddot{x} \cos \theta_{X} \cos \theta_{Y}+m g l \sin \theta_{X} \cos \theta_{Y} \\
& -2 m l^{2} \dot{\theta}_{X} \dot{\theta}_{Y} \sin \theta_{Y} \cos \theta_{Y}=0 \\
& m l^{2} \ddot{\theta}_{Y}+\ddot{y} m l \cos \theta_{Y}+m g l \sin \theta_{Y} \cos \theta_{X} \\
& -m l \ddot{x} \sin \theta_{X} \sin \theta_{Y}+m l^{2} \dot{\theta}_{X}^{2} \cos \theta_{Y} \sin \theta_{Y}=0
\end{aligned}
$$

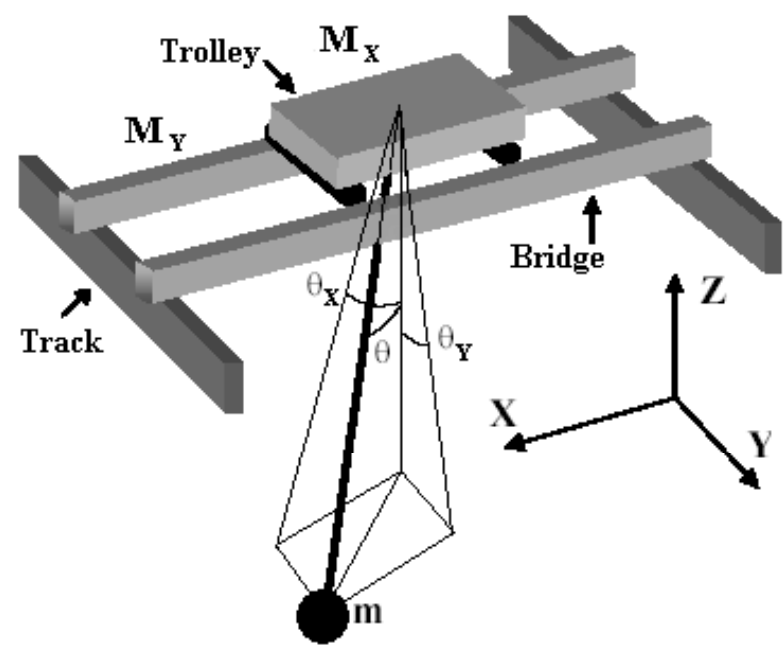

Figure 1 Two-Dimension Overhead Crane

where $D_{X}$ and $D_{Y}$ respectively denote the viscous damping coefficients of the crane in the $\mathrm{X}$ and $\mathrm{Y}$ directions, $f_{X}$ and $f_{Y}$ are the external forces on the overhead crane in the $\mathrm{X}$ and $\mathrm{Y}$ directions, respectively.

\section{System Model Analysi}

In industry, the maximum acceleration of the overhead crane is set smaller than the gravitational acceleration. For safety considerations, the rope length is usually kept constant when the overhead crane is in motion. For small swing around the vertical equilibrium, $\sin \theta_{X} \approx \theta_{X}$, $\sin \theta_{Y} \approx \theta_{Y}, \cos \theta_{X} \approx 1$ and $\cos \theta_{Y} \approx 1$. In addition, $\sin \theta_{X} \sin \theta_{Y} \approx 0, \dot{\theta}_{X}^{2} \approx 0, \dot{\theta}_{Y}^{2} \approx 0$ and $\dot{\theta}_{X} \dot{\theta}_{Y} \approx 0$ also hold for small swing. The nonlinear model can be simplified to the following linearized model:

$$
\begin{aligned}
& \left(M_{X}+m\right) \ddot{x}+m l \ddot{\theta}_{X}=f_{X}-D_{X} \dot{x} \\
& l \ddot{\theta}_{X}+\ddot{x}+g \theta_{X}=0 \\
& \left(M_{Y}+m\right) \ddot{y}+m l \ddot{\theta}_{Y}=f_{Y}-D_{y} \dot{y} \\
& l \ddot{\theta}_{Y}+\ddot{y}+g \theta_{Y}=0
\end{aligned}
$$

In practice, the crane is normally driven by servo-motors. The servomotor has three control modes: position control, speed control and torque control. In order to simplify the system dynamic model, the speed control mode is used. Through the following state feedback transformations:

$$
\begin{aligned}
& u_{X}=\left(M_{X}+m\right)^{-1}\left(f_{X}-D_{X} \dot{x}-m l \ddot{\theta}_{X}\right) \\
& u_{Y}=\left(M_{Y}+m\right)^{-1}\left(f_{Y}-D_{Y} \dot{y}-m l \ddot{\theta}_{Y}\right)
\end{aligned}
$$

the system model can be described as

$$
\begin{aligned}
& \ddot{x}=u_{X} \\
& l \ddot{\theta}_{X}+u_{X}+g \theta_{X}=0 \\
& \ddot{y}=u_{Y} \\
& l \ddot{\theta}_{Y}+u_{Y}+g \theta_{Y}=0
\end{aligned}
$$


The linearized dynamic model consists of the $\mathrm{X}$-direction transport dynamics and $\mathrm{Y}$-direction transport dynamics. The $\mathrm{X}$-direction dynamics and Y-direction dynamics are decouped and with the same structure. Therefore, the same control algorithm can be designed for both the $\mathrm{X}$ and $\mathrm{Y}$ direction transport systems. For this system model, the system control input is the acceleration of the overhead crane.

Because the viscous damping and the masses of trolleys and payload maybe are not known and the above transform makes the model be independent of them, the system control is easily implemented in practice.

\section{CONTROL DESIGN}

In this section, an adaptive slide mode fuzzy controller will be designed for the two-dimensional overhead crane. Assume the desired state is generalized coordinates origin. Since the two-dimensional overhead crane can be decouped into two independent transport systems, a control algorithm will be designed for both of them. Only the X-direction transport system is considered below.

\section{A. Sliding mode fuzzy control (SMFC)}

Consider a second-order system of the form as follows:

$$
\begin{aligned}
& \dot{x}_{1}=x_{2} \\
& \dot{x}_{2}=f(X)+b(X) u
\end{aligned}
$$

where, $X=\left(x_{1}, x_{2}\right)$ is state variable vector, $f(X)$ and $b(X)$ are continuous linear or nonlinear functions, $u$ is the control input. A sliding mode function can be defined as

$$
s=x_{2}+\lambda_{1} x_{1}
$$

Very similar to sliding mode control with boundary layer, the control input on the two sides of the sliding mode surface are opposite in sign and its magnitude is proportional to the distance between the state vector and the sliding mode surface. Therefore, the sliding mode fuzzy control is designed to:

\section{$R_{i}$ : IF $s$ IS $F_{i}$ THEN $u$ IS $U_{i}$}

where $F_{i}$ is the linguistic value of $s$ in the $i$ th-fuzzy rule, and $U_{i}$ is the linguistic value of $u$ in the $i$ th-fuzzy rule. The fuzzification of the sliding mode function is illustrated in Fig.2

\section{B. Adaptive sliding mode fuzzy control (ASMFC)}

The $\mathrm{X}$-direction transport systems can be represented as:

$$
\begin{aligned}
& \dot{x}_{1}=x_{2} \\
& \dot{x}_{2}=f_{1}(X)+b_{1}(X) u \\
& \dot{x}_{3}=x_{4} \\
& \dot{x}_{4}=f_{2}(X)+b_{2}(X) u
\end{aligned}
$$

where, $X=\left(x_{1}, x_{2}, x_{3}, x_{4}\right)$ is the state variable vector that include crane position, velocity, payload swing angle and angular velocity, $f_{1}(X), f_{1}(X), b_{1}(X)$ and $b_{2}(X)$ are continuous nonlinear functions, $u$ is the control input.

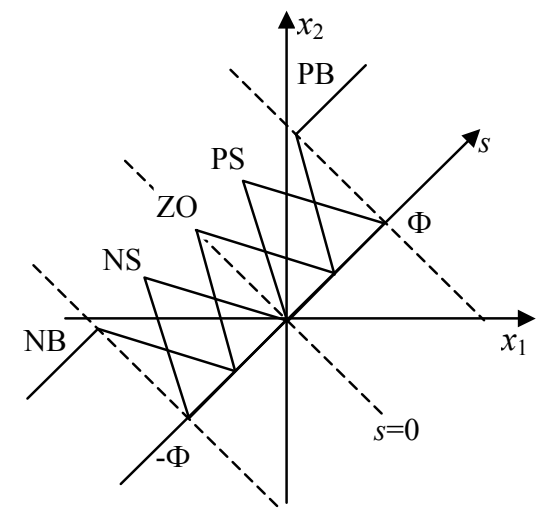

Figure 2 Fuzzification of sliding mode function in sliding mode fuzzy control

From (13), the X-direction transport system has two coupled subsystems: positioning subsystem and anti-swing subsystem. In order to decouple the system, two sliding mode functions are defined for the two subsystems:

$$
\begin{aligned}
& s_{1}=x_{2}+\lambda_{1} x_{1} \\
& s_{2}=x_{4}+\lambda_{2} x_{3}
\end{aligned}
$$

where $\lambda_{1}$ and $\lambda_{2}$ are positive real numbers.

System performance is very sensitive to the slope $\lambda_{1}$ (or $\lambda_{2}$ ) of the sliding mode function: when the value of $\lambda_{1}$ (or $\lambda_{2}$ ) becomes larger, the rise-time will become smaller, but at the same time, both overshoot and tuning-time will become larger, and vice versa. So a law is designed to adjust the slope $\lambda_{1}$ (or $\lambda_{2}$ ) of the sliding mode function in real time: when system state errors are large, a bigger slope of the sliding mode function is used in order to make the system state approach the sliding mode surface and equilibrium point. This is because the convergence speed on the sliding mode surface is high if a large $\lambda_{1}$ (or $\lambda_{2}$ ) is used. In mechanical systems, the value of $\lambda_{1}$ (or $\lambda_{2}$ ) is typically limited by three factors: the frequency of the lowest unmodelled structural mode, the largest unmodelled time delay, and the sampling rate.

According to the mechanical system limitation, the slope of the sliding mode function is given for the $l$ th subsystem $(l=1$ or 2$)$ by

$$
\lambda_{l}=\lambda_{l}^{b}+B_{l} \Delta \lambda_{l}
$$

where, $\lambda_{l}^{b}$ is the basic value of $\lambda_{l}, B_{l}$ is the tuning scope of $\lambda_{l}$, and $\Delta \lambda_{l}$ is the tuning variable. The value of $\Delta \lambda_{l}$ can be obtained according to the following fuzzy rules:

$$
R_{i} \text { : IF }\left|x_{2 l-1}\right| \text { IS } A_{l}^{i} \text { THEN } \Delta \lambda_{l} \text { IS } \Delta \lambda_{l}^{i}
$$

where, $R_{i}$ is the $i$ th item of $m$ rules, $A_{l}{ }^{i}$ is a fuzzy set of input variable $\left|x_{2 l-1}\right|$, and $\Delta \lambda_{l}^{i}$ is a fuzzy set of output variable $\Delta \lambda_{l}$. The output singleton fuzzy sets and the center-of-gravity 
defuzzification method are used:

$$
\Delta \lambda_{l}=\left(\sum_{i=1}^{m} \mu_{A_{l}^{i}}\left(\left|x_{2 l-1}\right|\right) \times \Delta \lambda_{l)}^{i}\right) / \sum_{i=1}^{m} \mu_{A_{l}^{i}}\left(\left|x_{2 l-1}\right|\right)
$$

$\mu_{A_{l}^{i}}\left(\left|x_{2 l-1}\right|\right)$ is the firing degree of the $i$ th rule.

The composite sliding mode function can be defined as

$$
s=s_{1}+\lambda s_{2}=x_{2}+\lambda_{1} x_{1}+\lambda\left(x_{4}+\lambda_{2} x_{3}\right)
$$

where $\lambda$ is a negative real number. Tuning the coefficient $\lambda$ can adjust the function of the positioning subsystem and the anti-swing subsystem on the sliding mode function. When $\lambda$ becomes smaller, the positioning subsystem is strengthened; and when $\lambda$ becomes larger, the anti-swing subsystem is strengthened. So another fuzzy inference is designed: if system state is far from sliding mode surface $s_{2}=0$, a larger value of $\lambda$ is adopted, vice versa. As the tuning of the sliding mode function slope, let

$$
\lambda=\lambda^{b}+B \Delta \lambda
$$

where, $\lambda^{b}$ is the basic value of $\lambda, B$ is the tuning scope of $\lambda$, and $\Delta \lambda$ is the tuning variable. The value of $\Delta \lambda$ can be obtained according to the following fuzzy rules:

$$
R_{j} \text { : IF }\left|s_{2}\right| \text { IS } F_{2}^{j} \text { THEN } \Delta \lambda \text { IS } \Delta \lambda_{j}
$$

where, $R_{j}$ is the $j$ th item of $n$ rules, $F_{2}{ }^{j}$ is a fuzzy set of input variable $\left|s_{2}\right|$, and $\Delta \lambda_{j}$ is a fuzzy set of output variable $\Delta \lambda$. The output singleton fuzzy sets and the center-of-gravity defuzzification method are used:

$$
\Delta \lambda=\left(\sum_{j=1}^{n} \mu_{F_{2}^{j}}\left(\left|s_{2}\right|\right) \times \Delta \lambda^{j}\right) / \sum_{j=1}^{n} \mu_{F_{2}^{j}}\left(\left|s_{2}\right|\right)
$$

where $\mu_{F_{2}^{j}}\left(\left|s_{2}\right|\right)$ is the firing degree of the $j$ th rule.

Only in the anti-swing subsystem of the X-direction transport system, the slope of the sliding mode function is automatically adjusted by fuzzy inference system, which is called as adjustor 1 . The relationship between the positioning subsystem and anti-swing subsystem are automatically tuned by another fuzzy inference system, which is called as adjustor 2. For the X-direction transport system of the overhead crane, the composite sliding mode function $\mathrm{s}$ works as the input to the sliding mode fuzzy control. The fuzzy rules are:

$$
R_{k} \text { : IF s IS } F_{k} \text { THEN } u_{f} \text { IS } U^{k}
$$

where, $R_{k}$ is the $k$ th item of $p$ rules, $F^{k}$ is a fuzzy set of input variable $s$, and $U^{k}$ is a fuzzy set of output variable $u_{f}$. The output singleton fuzzy sets and the center-of-gravity defuzzification are used:

$$
u_{f}=\left(\sum_{k=1}^{p} \mu_{F^{k}}(s) \times U^{k}\right) / \sum_{k=1}^{p} \mu_{F^{k}}(s)
$$

where $\mu_{F^{k}}(s)$ is the firing degree of the $j$ th rule and $u_{f}$ is the output of the adaptive sliding mode fuzzy controller.

Now consider the reaching condition of the sliding mode surface that is based on the flowing Lyapunov function:

$$
V=s^{2} / 2
$$

By taking the time derivative of equation (18) and substituting into equation (22), it is easy to obtain from equation (13)

$$
\begin{aligned}
s \dot{s}= & s\left(f_{1}(X)+\lambda f_{2}(X)+\lambda_{1} x_{2}+\lambda \lambda_{2} x_{4}\right) \\
& +\left(b_{1}(X)+\lambda \lambda_{2} b_{2}(X)\right) s u
\end{aligned}
$$

Form equation (9), we can see: $b_{1}(X)$ and $\lambda_{2}$ is positive, and $b_{2}(X)$ is negative. When $\lambda$ is negative, the term in equation (23), $b_{1}(X)+\lambda b_{2}(X)$ are always positive. Therefore, increasing the control input $u$ will result in decreasing $s \dot{s}$ as the sliding mode function $s$ is negative, and decreasing the control input $u$ will result in decreasing $s \dot{s}$ as the sliding mode function $s$ is positive.

Remark 1: According to the system dynamic model that the X-direction transport system and Y-direction transport system are decoupled and with the same linearized model, the control algorithm is also applicable to Y-direction transport system.

Remark 2: The proposed control design is independence of the linearized system model, i.e. the control algorithm is designed for nonlinear overhead crane system. The linearized model derived in section 2 is used to explain the two-dimensional overhead crane consisting of two approximately independent transport systems.

Remark 3: When the initial payload angles are zeros, both $\mathrm{X}$-direction and $\mathrm{Y}$-direction transportations will arrive at the goal at the same time with the same adaptive sliding mode fuzzy controller.

Remark 4: Now that the servomotors adopt speed control mode while the output of the controller is acceleration, actual input of the servomotors is:

$$
u=\int_{t_{0}}^{t_{c}} u_{f} d t
$$

where $t_{0}$ and $t_{c}$ are initial time and current time separately.

The control scheme is illustrated in figure 3.

\section{EXPERIMENT RESULTS}

To confirm the effectiveness of the proposed control algorithm, some experiments have been performed with a two-dimensional prototype overhead crane illustrated in Figure 4. The prototype consists of two sets of components that include mechanical system, data sampling system, and control system. For the mechanical system, the trolleys are driven by AC servomotors, and the payload is connected to a cable that is attached to the underside of the trolley, where two precise angle sensors are installed to measure the swing angles of X-direction and $\mathrm{Y}$-direction. The control algorithm is implemented on a Pentium III $800 \mathrm{MHz}$ PC running under the Windows operating system.

In the control algorithm, the adaptive tuning of the slope of the sliding mode function is only used for the anti-swing 
subsystems. The parameters of the controller are as follows: $\lambda_{1}=0.5, \lambda_{2}^{b}=2.28, B_{2}=3, \lambda^{b}=-1, B=-4.6$. The tunings of $\lambda$ and $\lambda_{2}$ adopt the same fuzzy rules table, given in table 1 . The sliding mode fuzzy rules are provided in table 2 .

Table 1 Rules table for tuning $\lambda$ and $\lambda_{2}$

\begin{tabular}{llrl}
\hline$\left|e_{3}\right|$ or $\left|s_{2}\right|$ & $\mathrm{S}$ & $\mathrm{M}$ & $\mathrm{L}$ \\
\hline$\lambda_{2}$ or $\Delta \lambda$ & 0 & 0.5 & 1 \\
\hline
\end{tabular}

Table 2 Rules table of sliding mode fuzzy controller

\begin{tabular}{lrrccc}
\hline$s$ & NB & NS & ZO & PS & PB \\
\hline$u_{f}$ & 2.5 & 1.25 & 0 & -1.25 & -2.5 \\
\hline
\end{tabular}

Figure 5 and 6 show the experimental results of transport from position $(-1.2,-0.6)$ to position $(0,0)$ with zero initial angles. Figure 7 shows the X-direction transport when the initial angle is not zero. In the above figures, velocity is $0.2 \mathrm{~m} / \mathrm{s}$ each grid in $\mathrm{Y}$-axis. Figure 8,9 and 10 show the damping swing experiment results and the control is added from the 6 th second. Figure 8 is angle phase plane and figure 9 and 10 are angles time responses. From the experiment results, it is clear that the control law can make the $\mathrm{X}$-direction and Y-direction transports arrive at goal at the same time when initial angle is zero, and the control law can damp swing angle at goal whether the initial angle is zero. Moreover, the control law can damp swing angle in short time while keeping position.

\section{CONCLUSION}

In this paper, a two-dimensional overhead crane is transformed to two independent systems that are with the same dynamic model in order to simplify the controller design. An adaptive sliding mode fuzzy control approach has been designed for both $\mathrm{X}$-direction transportation and Y-direction transportation, and its effectiveness has been demonstrated by experiments on a two-dimensional prototype overhead crane. The experiments have shown the proposed control law guarantees both accurate positioning control and prompt damping of payload swing. The stability and performance of the proposed control law are guaranteed in spite of large initial swing angle.

\section{REFERENCES}

[1] Bae-Jeong Park, Keum-Shik Hong, Chang-Do Huh, "Time-Efficient Input Shaping Control of Container Crane Systems", in Proc. Of IEEE Int. Conf. on Control, 2000, pp.80-85.

[2] William singhose, Lisa Porter, Michael Kenison etc, "Effects of Hoisting on the Input Shaping Control of Gantry Cranes", Control Engineering Practice, vol. 8, pp.1159-1165, 2000.

[3] Ho-Hoon Lee, "Modeling and Control of a Three-Dimensional Overhead Crane", J. of Dynamic System, Measurement, and Control, vol. 120, pp.471-476, 1998.

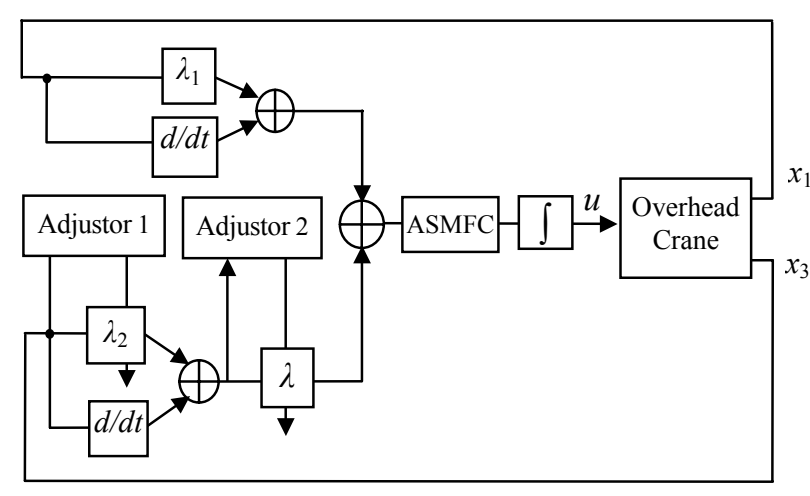

Figure 3 Control Scheme

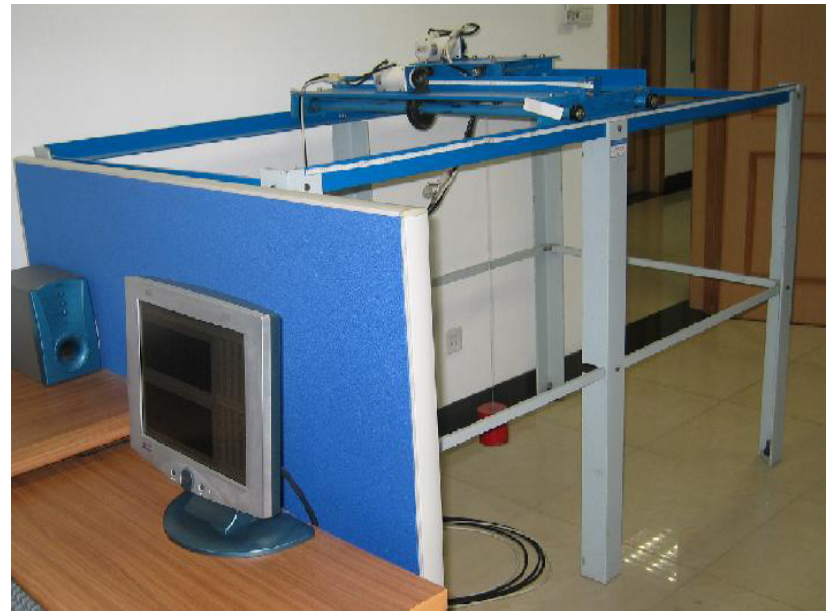

Figure 4 Two-dimension Prototype Overhead Crane

[4] Alessandro Giua, Carla Seatzu, Giampaolo Usai, "Observer-controller Design for Cranes via Lyapunov Equivalence”, Automatica, vol. 35, pp. 669-678, 1999.

[5] Ho-Hoon Lee and Sung-Kun Cho, "A New Fuzzy-Logic Anti-Swing Control for Industrial Three-Dimensional Overhead Cranes", in Proc. of IEEE int. Conf. on Robotics \& Automation, 2001, pp. 2956-2961.

[6] Keqiang Hua, "Fuzzy Anti-swing Technology for Overhead Crane", $J$. of civil aviation university of China, vol. 3, 2000, pp.12-23.

[7] Michael J.Nalley and Mohamed B.Trabia, "Control of Overhead Cranes Using a Fuzzy Logic Controller", J. of Intelligent and Fuzzy System, vol. 8, pp.1-18, 2000.

[8] M. J. Er, M. Zribi and K. L. Lee, "Variable Structure Control of an Overhead Crane", in Proc. of IEEE Int. Conf. on Control Application, 1998, pp.398-402.

[9] Mansour, A.Kakoub and Mohamed Zribi, "Robust Control Schemes for an Overhead Crane", J. of Vibration and Control, vol. 7, pp.395-416, 2001.

[10] M. Hasanul Basher, "Swing-free Transport Using Variable Structure Model Reference Control", in Proc. of IEEE Southeastcon. 2001, pp.85-92.

[11] Y. Fang, W. E. Dixon, D. M. Dawson and E. Zergeroglu, "Nonlinear Coupling Control Laws for a 3-DOF Overhead Crane System", in Proc. of IEEE Conf. on Decision and Control, 2001, pp. .3766-3771 


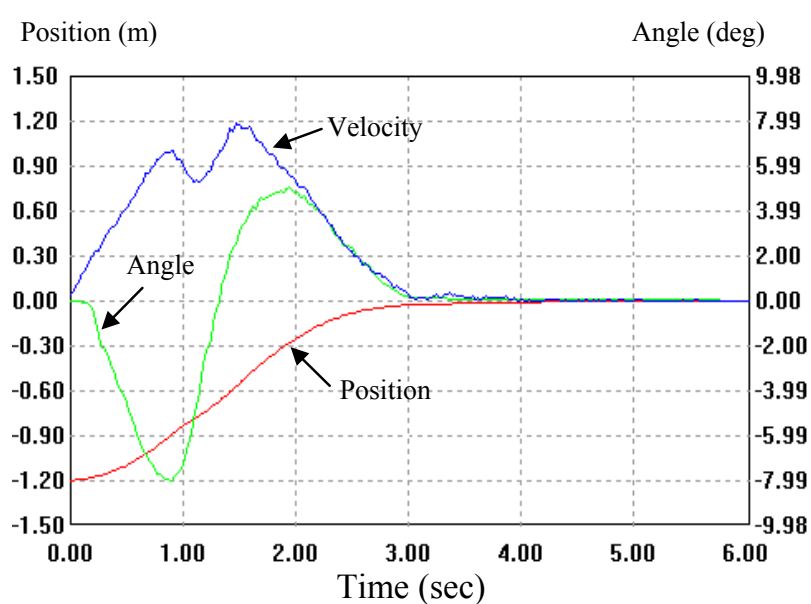

Figure 5 Time Responses of X-direction Transport

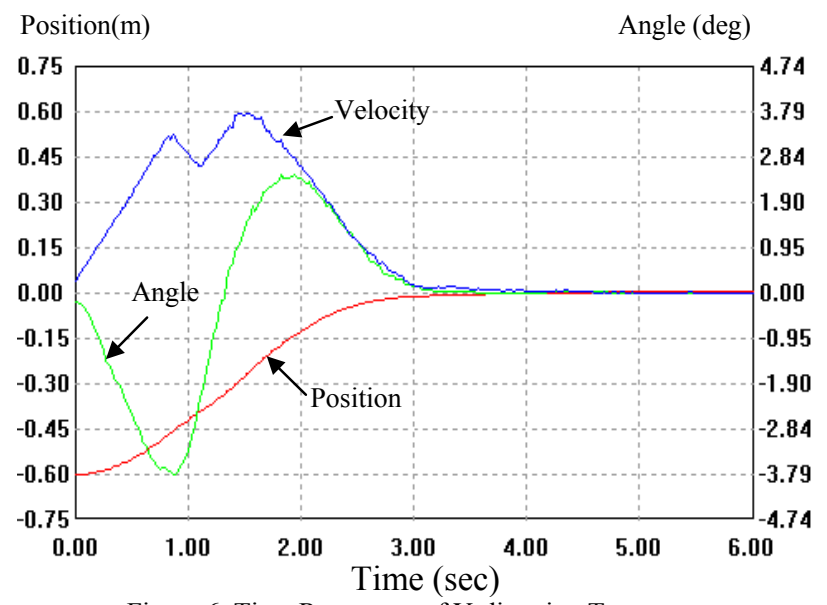

Figure 6 Time Responses of Y-direction Transport

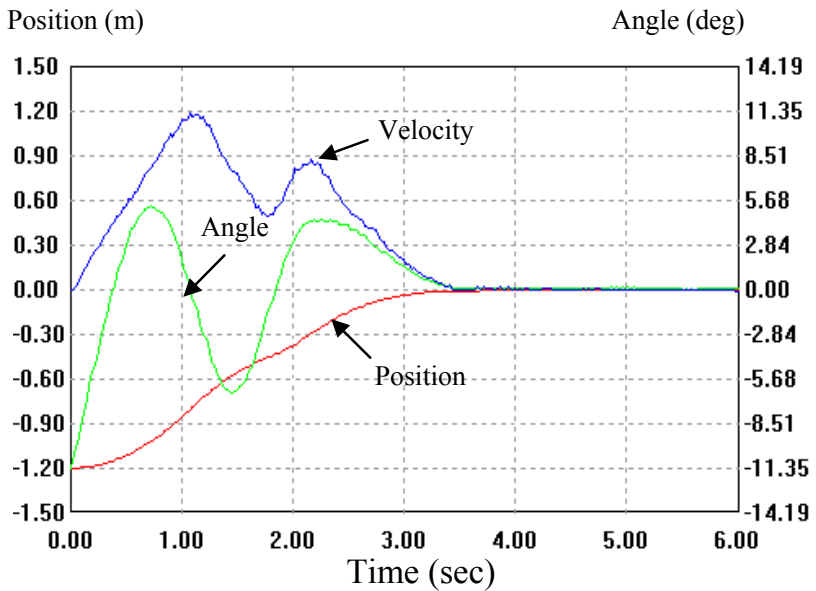

Figure 7 Time Responses with Initial Angle

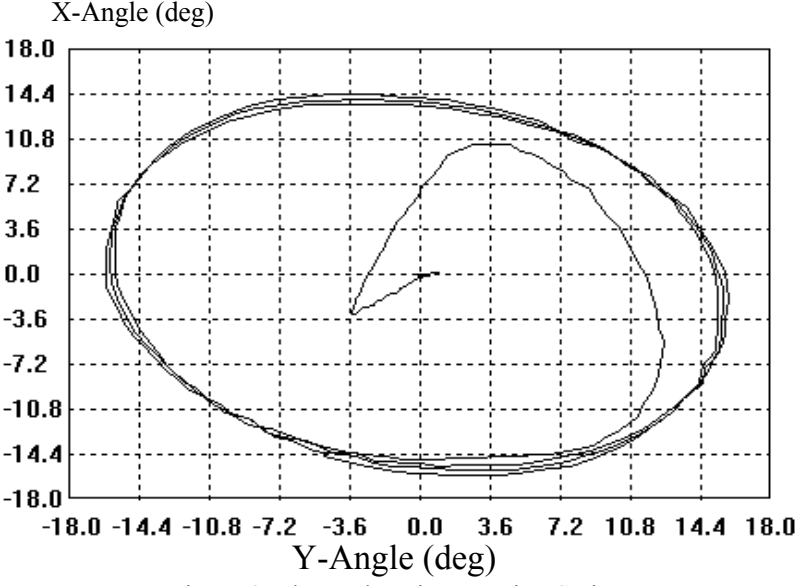

Figure 8 Phase Plane in Damping Swing

X-Angle (deg)

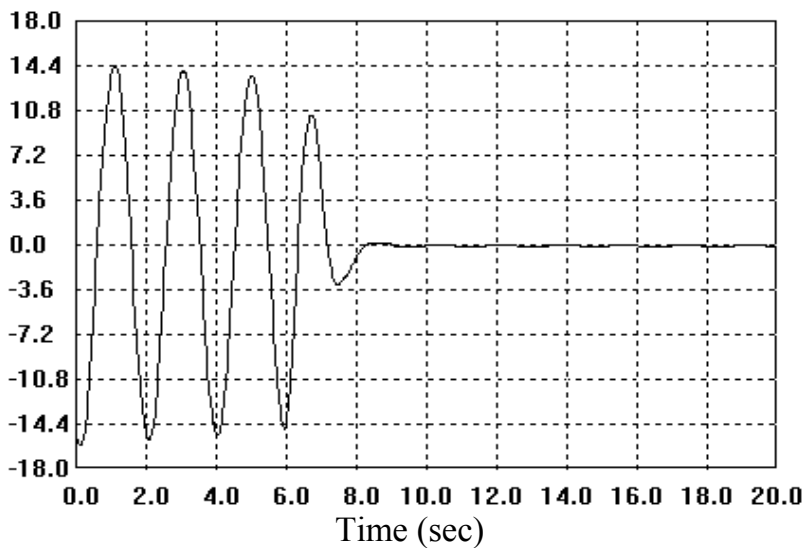

Figure 9 Time Response of X-direction Angle Damping

Y-Angle (deg)

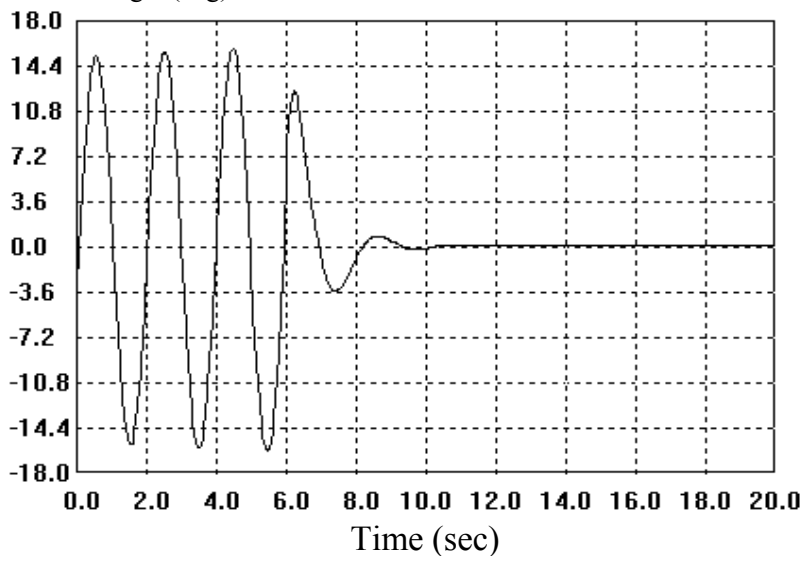

Figure 10 Time Response of Y-direction Angle Damping 